\title{
PerformanCE ANALYSIS Of S-ALOHA USED AS A RANDOM ACCES CHANNEL ON MOBILE COMMUNICATIONS
}

\author{
D. Covarrubias', A. Mendez², \& C. Vargas ${ }^{3}$ \\ ${ }^{1}$ CICESE Research Center, Km. 107 Carretera Tijuana-Ensenada \\ Ensenada, Baja California, 22860 México, \\ Phone: (52) 646.175.0555, Fax: (52) 646.175.0554 \\ e-mail: dacoro@cicese.mx \\ ${ }^{2}$ Autonomous University of Tamaulipas, UAT-UAMRR \\ Carretera Reynosa-San Fernando, Reynosa, Tamaulipas, 88779 MEXICO \\ Phone: (52) 899.921.3300, Fax: (52) 899.921.3301 \\ e-mail: almendez@uat.edu.mx

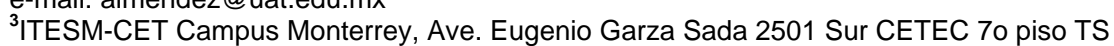 \\ Monterrey, Nuevo León, 64849 México \\ Phone (52) 818.158.2081, Fax: (52) 818.359.7211 \\ e-mail: cvargas@itesm.mx
}

Received: December $10^{\text {th }}$, 2002. Accepted: February $24^{\text {th }}, 2005$

ABSTRACT

This paper evaluates the effects of Rayleigh fading, shadowing, and spatial distribution of the Mobile Terminals (MTs) on the operating performance of Slotted Aloha (S-Aloha) used as a Random Access Channel (RACH). This paper also evaluates the capture probability in a channel exhibiting Rayleigh fading, shadowing, and the effect of spatial distribution of the MTs. In addition, this work presents a different version of the steady-sate probabilities of the Markov chain of S-Aloha considering capture effect. This article simulates the stabilization of S-Aloha by utilizing an algorithm that dynamically controls the retransmission probabilities. Finally, this paper presents a numerical analysis of these simulations and identifies the operating of parameters that have shown to degrade system response.

\section{RESUMEN}

Este artículo evalúa las prestaciones de Aloha ranurado (S-Aloha), usado por el terminal móvil en la fase de petición de canal aleatoria, considerando los efectos de desvanecimiento Rayleigh, sombreo y distribución de los terminales móviles. También evalúa la probabilidad de captura en un canal exhibiendo desvanecimiento Rayleigh, sombreo y distribución espacial de los móviles. Además, se presenta una versión diferente de las probabilidades en estado estable con efecto captura de la cadena de Markov de S-Aloha. Por otro lado, se simula la estabilización de S-Aloha, empleando un algoritmo que controla dinámicamente las probabilidades de retransmisión. Por último, se lleva a cabo un análisis numérico de los resultados y se identifican aquellos parámetros que degradan la respuesta del sistema de comunicaciones móviles celulares.

KEYWORDS: Mobile communications, Capture effect, Retransmission algorithm, S-Aloha stability.

1. INTRODUCTION

In mobile communication systems, a Medium Access Control (MAC) protocol facilitates communication between the Mobile Terminal (MT) and Base Station (BS) beginning with a MT service request via the Random Access Channel $(\mathrm{RACH})$. It is worth to emphasize that given the present randomness characteristics in a mobile communication 
scenario, it is necessary to count with a Medium Access Control technique, that can efficiently cope with the possible interference among MTs which use simultaneously the RACH directed toward the same BS, i.e., the MAC technique should help prevent and solve these problems, as well as optimize the request of the channel in order to have high throughput and low average delay.

Due to Rayleigh fading, shadowing, and spatial distribution of the MTs, the BS typically receives different power levels from each MT. Because of these differing power levels, the S-Aloha access protocol capture effect will likely enable one data packet out of a large number of colliding data packets to be read correctly, thus improving S-Aloha throughput [1, 2].

It is well known that S-Aloha (as a RACH) has serious limitations in terms of throughput (0.368 is the maximum), and has stability problems as channel traffic (or load channel) increases [3]. When using a retransmission algorithm such as S-Aloha, it is necessary to base that algorithm on the dynamic control of retransmission probabilities.

This paper presents a performance evaluation of S-Aloha determining what parameters are highly sensitive to system performance. Section 2 of this paper offers a different opinion analyzed in [4, 5], presenting an alternative version of the state transition probabilities of a cellular system while obtaining the capture probability of a channel exhibiting Rayleigh fading, shadowing, and spatial distribution of the MTs. This section also models the S-Aloha access protocol using Markov chains to provide a detailed insight into the dynamic behavior of the network. In addition, this section presents an algorithm of retransmission adaptable to the conditions of traffic changes.

Section 3 of this paper simulates the capture effect and the stabilization of the system. This simulation process utilizes a Real Time Emulator (RTE) [6], to enhance the simulation of Rayleigh fading, shadowing, and spatial distribution of the MTs to enable performance testing of a cellular system under various scenarios. Finally, this paper presents a numerical analysis of these simulations and identifies parameters that have shown to degrade system performance.

\section{MODELING OF THE RACH}

The RACH analysis presented in this paper considers only the up-link communication (from MT to BS). A retransmission occurs when there is a collision and the MT receives a BS positive acknowledgment. As mentioned before the first interaction between the MT and the BS is given in the access request, in which, two or more MTs try to transmit, or when retransmitting in the same time-slot, a collision of request packets unavoidably is presented. Once reached this situation, the system puts the MTs involved in the collision in a backlogged state. In view of such possibility and given the randomness process it is necessary the modeling of the state probabilities. Due to the possibility to determine the number of backlogged MTs in each time-slot, by controlling the transmission probability of MTs in retransmission state (backlogged), as well as in idle state (new generated packets), an orderly arrival of requests to the RACH channel is permitted. Since MTs are identical and operate independently, the behavior of S-Aloha is described by means of a finite state Markov chain with $M$ mobile terminals modeled by a birth-death process.

The initial stage of RACH modeling and analysis is performed on a slot-by-slot basis, where the time-slots are numbered sequentially, $k=0,1,2, \ldots$. Moreover, let $\eta_{s}(k)$ be the number of backlogged MTs at the beginning of the $k$-th slot. The quantity $\eta_{s}(k)$ can assumes one of $(M+1)$ possible values of $\{0,1,2, \ldots, M\}$ and can be considered as the state variable of the system. Since the system is memoryless, $\eta_{s}(k)$ is a finite Markov chain with the transition probability $P\left(P\left[p_{i j}\right]\right)$ where $p_{i j}=\lim _{k \rightarrow \infty} \operatorname{Pr}\left(\eta_{s}(k)=j \mid \eta_{s}(k-1)=i\right)$ is given by 


$$
p_{i j}= \begin{cases}0, & j\langle i-1, \\
{\left[i v(1-v)^{i-1}\right](1-\varphi)^{M-i},} & j=i-1, \\
\left.1-i v(1-v)^{i-1}\right](1-\varphi)^{M-i}+\left[(M-i) \varphi(1-\varphi)^{M-i-1}\right](1-v)^{i}, & j=i, \\
(M-i) \varphi(1-\varphi)^{M-i-1}\left[1-(1-v)^{i}\right], & j=i+1, \\
{\left[\begin{array}{l}
M-i \\
j-i
\end{array}\right] \varphi^{j-i}(1-\varphi)^{M-j},} & j\rangle i+1,\end{cases}
$$

where $v$ is the probability that a MT retransmits a packet, and $\varphi$ is the probability that a MT generates a new packet.

For $0<\varphi<1$ and $0<v<1$, the Markov chain is finite, irreducible and aperiodic. Under these conditions, the Markov chain is always stable in the sense that at time, $\pi_{i}=\lim _{k \rightarrow \infty} \operatorname{Pr}\left(\eta_{s}(k)=i\right)$ converges to a valid probability mass function (i.e., all the probabilities are non-negative and they add to 1 ). The steady-state probability vector $\pi$, whose elements are $\pi_{i}$ is the solution to the finite set of linear equations $\boldsymbol{\pi}=\boldsymbol{\pi P}$, and $\sum_{i=0}^{M} \pi_{i}=1$, [7]. It is advisable to analyze the system behavior in a steady-state, since the solution to the distribution in equilibrium of the steady-state of the Markov chain enables the evaluation of the system performance.

\subsection{Modeling the Throughput of the RACH}

In order to evaluate the system throughput, it is assumed that the initiation of transmission is coincidental to the beginning of each time-slot, and that activity in any given time-slot is independent of the activity within any previous time-slot. Based on this premise, the fraction of time that a channel transports useful information or throughput, $S$, is equal to the average fraction of slots in any successful transmission.

The throughput $S$, is expressed as

$$
\begin{gathered}
S=E\left[P_{\text {succ }}(i)\right]=\sum_{i=0}^{M} P_{\text {succ }}(i) \cdot \pi_{i}, \\
P_{\text {succ }}(i)=(1-v)^{i} \cdot(M-i) \cdot \varphi \cdot(1-\varphi)^{M-i-1}+i \cdot v \cdot(1-v)^{i-1} \cdot(1-\varphi)^{M-i},
\end{gathered}
$$

where the vector of probability of the steady state $\pi$ can be calculated resolving the finite set of linear equations

$\pi=\pi P$.

\subsection{Modeling the Capture Effect of the RACH}

Up to this point, the modeling of S-Aloha as a RACH, has been considered within a noiseless channel where all data packets arrive at the BS with the same power levels. Under these conditions, when two or more data packets simultaneously arrive at the BS, they will collide and will be destroyed. However, since data packets typically arrive at the BS at different power levels, capture effect occurs and reduces the probability of the destruction of the colliding data packets, which results in an increase of system throughput [8].

Capture probability $P_{\text {capt }}(i), i>0$, is defined as the probability that one of $i$ collided data packets will be successfully received. In addition, the threshold between the power of the signal and the power of the possible interferers should be greater than a certain margin known this as capture ratio $(R)$ [1]. 
Considering this scheme, to deference from [4, 5], a differing version of the state transition probabilities regarding capture effect is determined by

$$
p_{i j}=\left\{\begin{array}{ll}
0, & j\langle i-1, \\
(1-\varphi)^{M-i} \sum_{c=1}^{i}\left(\begin{array}{l}
i \\
c
\end{array}\right) v^{c}(1-v)^{i-c} P_{\text {capt }}(c), & j=i-1, \\
1\{M>j\}\left(\begin{array}{l}
M-i \\
j-i+1
\end{array}\right) \varphi^{j-i+1}(1-\varphi)^{M-j-1} \sum_{k=0}^{i}\left(\begin{array}{l}
i \\
k
\end{array}\right) v^{k}(1-v)^{i-k} P_{\text {capt }}(k+j-i+1)+\left(\begin{array}{l}
M-i \\
j-i
\end{array}\right) \varphi^{j-i}(1-\varphi)^{M-j} & \\
\cdot \sum_{k=0}^{i}\left(\begin{array}{l}
i \\
k
\end{array}\right) v^{k}(1-v)^{i-k}\left[1-P_{\text {capt }}(k+j-i)\right], & M \geq j \geq i .
\end{array} .\right.
$$

Since the Markov chain is homogeneous, the vector of steady-state probability $\pi$, can be calculated by solving a group of non-linear equations $\pi=\pi \boldsymbol{P}$, and $\sum_{i=0}^{M} \pi_{i}=1$.

With these probabilities, the throughput performance can be computed by

$$
S=E\left[P_{\text {succ }}(i)\right]=\sum_{i=0}^{M} P_{\text {succ }}(i) \cdot \pi_{i}
$$

where the probability of success, $P_{\text {succ }}(i)$, considering the capture effect, is given by

$$
P_{\text {succ }}(i)=\sum_{r=0}^{M-i}\left(\begin{array}{l}
M-i \\
M-i-r
\end{array}\right) \varphi^{r}(1-\varphi)^{M-i-r} \sum_{c=0}^{i}\left(\begin{array}{l}
i \\
c
\end{array}\right) v^{c}(1-v)^{i-c} P_{\text {capt }}(c+r)
$$

According to equations (5) and (6), we need to determine the capture probabilities $P_{\text {capt }}$, while considering the spatial distribution of the MTs, and the presence of radio channel characterized by Rayleigh fading, and shadowing.

\subsection{Spatial Distribution of the Mobiles}

Another point to consider is to find the modeling of spatial distribution that best adjusts to the mobility of the MTs. According to [9], the difference between the performance of S-Aloha when considering uniform and non-uniform spatial distributions (particularly Bell-Shaped) are minimum (about 6\%), so given the ease of mathematical treatment we will use in our modeling a uniform distribution.

\subsection{Presence of the Radio Channel}

The radio channel can be characterized statistically, not only by noise, but also by groundwave propagation fading, and shadowing. We use a propagation model in which the normalized received signal power varies with the distance between the BS and the MT with a power loss factor $\alpha$ [10]. For mobile radio systems, a typical value of $\alpha$ is 4 [11].

The radio signal that is transmitted suffers fading caused by multipath and shadowing caused by obstacles, in which case the power of the signal can be characterized by a log-normal probability density function [10], with a standard deviation, $\sigma$, of $5 \mathrm{~dB}$ for an outdoor cellular environment. The fading of the instantaneous envelope of the received signal follows a Rayleigh pdf [12].

So according to [8], the expression for the probability of capture considering the uniform spatial distribution and the effect of the channel (Rayleigh + Shadowing) is given by 


$$
P_{\text {capt }}=B^{2} \cdot \int_{0}^{\infty} \int_{0}^{\infty} \int_{0}^{1} 2 y \cdot \frac{1}{\left(R y^{4} z+u\right) \cdot z} \cdot e^{-\frac{(A-\log z)^{2}}{2 \sigma^{2}}} \cdot e^{-\frac{(A-\log u)^{2}}{2 \sigma^{2}}} \cdot d y d u d z
$$

where ${ }_{B}=\frac{\log e}{\sqrt{2 \pi} \sigma}, A=\log W_{0}+\frac{\sigma^{2}}{2 \log e}, W_{0}$ is the mean value of the signal power, $\sigma$ is the standard deviation, $y$ is the distance between the MT and BS, log is the logarithm base 10 , and $R$ the capture ratio.

\subsection{Analysis of Stability of the RACH}

According to the feedback information of a slot (idle, success, and collision), we have modeled the algorithm of retransmission through Markov chains. Utilizing feedback information, the algorithm of retransmission has the capacity to execute channel load control, by performing channel load estimation, $\hat{n}$, at the beginning of each timeslot $[3,13]$.

Under these circumstances, each backlogged data packet is retransmitted with a probability $v\left(\begin{array}{l}\hat{n} \\ )\end{array}\right)=\min \left(1, \frac{1}{\hat{n}}\right) \cdot \operatorname{In}$ this algorithm, all the MTs observe the feedback channel. Thus, they obtain information about the outcome of each timeslot. In time-slot $k+1$, each MT independently performs an update of its estimate $\hat{n}$ and obtains an $\hat{n}_{k+1}$ depending on the outcome of the previous time-slot

$$
\hat{n}_{k+1}=\hat{n}+\left\{\begin{array}{cc}
e s t \_i, & \text { if slot } k \text { was idle, } \\
e s t \_s, & \text { f slot } k \text { had one success } \\
\text { (active state), } & \text { est_c }, \begin{array}{l}
\text { if slot } k \text { had one collision } \\
\text { (backlog state), }
\end{array}
\end{array}\right.
$$

where est_i, est_s, est_c are defined as:

$$
\begin{aligned}
& \text { est_i }=2-e, \\
& \text { est_ } s=2-\frac{e}{1-p_{e}}, \\
& \text { est_c }=2 .
\end{aligned}
$$

where $p_{e}$ is the probability of the packet not being accepted by the receiver.

With these values, it is possible to calculate in a dynamic form the estimate, Equation (8), obtaining control of the load of the system. Therefore, the objective is to operate with a binary exponential retransmission algorithm [14] that permits us to stabilize the response of S-Aloha, as it will be seen in Section 3.

\subsection{Modeling the Throughput of the RACH}

In order to evaluate the system throughput, it is assumed that the initiation of transmission is coincidental to the beginning of each time-slot, and that activity in any given time-slot is independent of the activity within any previous time-slot. Based on this premise, the fraction of time that a channel transports useful information or throughput, $S$, is equal to the average fraction of slots in any successful transmission.

The throughput $S$, is expressed as 


$$
\begin{gathered}
S=E\left[P_{\text {succ }}(i)\right]=\sum_{i=0}^{M} P_{\text {succ }}(i) \cdot \pi_{i}, \\
P_{\text {succ }}(i)=(1-v)^{i} \cdot(M-i) \cdot \varphi \cdot(1-\varphi)^{M-i-1}+i \cdot v \cdot(1-v)^{i-1} \cdot(1-\varphi)^{M-i},
\end{gathered}
$$

where the vector of probability of the steady state $\pi$ can be calculated resolving the finite set of linear equations $\pi=\pi P$.

\subsection{Modeling the Capture Effect of the RACH}

Up to this point, the modeling of S-Aloha as a RACH, has been considered within a noiseless channel where all data packets arrive at the BS with the same power levels. Under these conditions, when two or more data packets simultaneously arrive at the BS, they will collide and will be destroyed. However, since data packets typically arrive at the BS at different power levels, capture effect occurs and reduces the probability of the destruction of the colliding data packets, which results in an increase of system throughput [8].

Capture probability $P_{\text {capt }}(i), i>0$, is defined as the probability that one of $i$ collided data packets will be successfully received. In addition, the threshold between the power of the signal and the power of the possible interferers should be greater than a certain margin known this as capture ratio $(R)[1]$.

Considering this scheme, to deference from $[4,5]$, a differing version of the state transition probabilities regarding capture effect is determined by

$$
p_{i j}=\left\{\begin{array}{ll}
0, & j\langle i-1, \\
(1-\varphi)^{M-i} \sum_{c=1}^{i}\left(\begin{array}{l}
i \\
c
\end{array}\right) v^{c}(1-v)^{i-c} P_{\text {capt }}(c), & j=i-1, \\
1\{M>j\}\left(\begin{array}{l}
M-i \\
j-i+1
\end{array}\right) \varphi^{j-i+1}(1-\varphi)^{M-j-1} \sum_{k=0}^{i}\left(\begin{array}{l}
i \\
k
\end{array}\right) v^{k}(1-v)^{i-k} P_{\text {capt }}(k+j-i+1)+\left(\begin{array}{c}
M-i \\
j-i
\end{array}\right) \varphi^{j-i}(1-\varphi)^{M-j} & \\
\cdot \sum_{k=0}^{i}\left(\begin{array}{l}
i \\
k
\end{array}\right) v^{k}(1-v)^{i-k}\left[1-P_{\text {capt }}(k+j-i)\right], & M \geq j \geq i .
\end{array} .\right.
$$

Since the Markov chain is homogeneous, the vector of steady-state probability $\pi$, can be calculated by solving a group of non-linear equations $\pi=\pi \boldsymbol{P}$, and $\sum_{i=0}^{M} \pi_{i}=1$.

With these probabilities, the throughput performance can be computed by

$$
S=E\left[P_{\text {succ }}(i)\right]=\sum_{i=0}^{M} P_{\text {succ }}(i) \cdot \pi_{i},
$$

where the probability of success, $P_{\text {sucd }}(\lambda)$, considering the capture effect, is given by

$$
P_{\text {succ }}(i)=\sum_{r=0}^{M-i}\left(\begin{array}{l}
M-i \\
M-i-r
\end{array}\right) \varphi^{r}(1-\varphi)^{M-i-r} \sum_{c=0}^{i}\left(\begin{array}{l}
i \\
c
\end{array}\right) v^{c}(1-v)^{i-c} P_{\text {capt }}(c+r) .
$$


According to equations (5) and (6), we need to determine the capture probabilities $P_{a x p}$, while considering the spatial distribution of the MTs, and the presence of radio channel characterized by Rayleigh fading, and shadowing.

\subsection{Spatial Distribution of the Móviles}

Another point to consider is to find the modeling of spatial distribution that best adjusts to the mobility of the MTs. According to [9], the difference between the performance of S-Aloha when considering uniform and non-uniform spatial distributions (particularly Bell-Shaped) are minimum (about 6\%), so given the ease of mathematical treatment we will use in our modeling a uniform distribution.

\subsection{Presence of the Radio Channel}

The radio channel can be characterized statistically, not only by noise, but also by groundwave propagation fading, and shadowing. We use a propagation model in which the normalized received signal power varies with the distance between the BS and the MT with a power loss factor $\alpha$ [10]. For mobile radio systems, a typical value of $\alpha$ is 4 [11].

The radio signal that is transmitted suffers fading caused by multipath and shadowing caused by obstacles, in which case the power of the signal can be characterized by a log-normal probability density function [10], with a standard deviation, $\sigma$, of $5 \mathrm{~dB}$ for an outdoor cellular environment. The fading of the instantaneous envelope of the received signal follows a Rayleigh pdf [12].

So according to [8], the expression for the probability of capture considering the uniform spatial distribution and the effect of the channel (Rayleigh + Shadowing) is given by

$$
P_{\text {capt }}=B^{2} \cdot \iint_{0}^{\infty} \iint \frac{1}{0} 2 y \cdot \frac{1}{\left(R y^{4} z+u\right) \cdot z} \cdot e^{-\frac{(A-\log z)^{2}}{2 \sigma^{2}}} \cdot e^{-\frac{(A-\log u)^{2}}{2 \sigma^{2}}} \cdot d y d u d z
$$

where ${ }_{B}=\frac{\log e}{\sqrt{2 \pi \sigma}}, A=\log W_{0}+\frac{\sigma^{2}}{2 \log e}, W_{0}$ is the mean value of the signal power, $\sigma$ is the standard deviation, $y$ is the distance between the MT and $\mathrm{BS}$, log is the logarithm base 10 , and $R$ the capture ratio.

\subsection{Analysis of Stability of the RACH}

According to the feedback information of a slot (idle, success, and collision), we have modeled the algorithm of retransmission through Markov chains. Utilizing feedback information, the algorithm of retransmission has the capacity to execute channel load control, by performing channel load estimation, $\hat{n}$, at the beginning of each timeslot $[3,13]$.

Under these circumstances, each backlogged data packet is retransmitted with a probability $v(\hat{n})=\min \left(1, \frac{1}{\hat{n}}\right) \cdot$ In this algorithm, all the MTs observe the feedback channel. Thus, they obtain information about the outcome of each timeslot. In time-slot $k+1$, each MT independently performs an update of its estimate $\hat{n}$ and obtains an $\hat{n}_{k+1}$ depending on the outcome of the previous time-slot 


$$
\hat{n}_{k+1}=\hat{n}+\left\{\begin{array}{cc}
e s t \_i, & \text { if slot } k \text { was idle, } \\
e s t \_s, & \text { if slot } k \text { had one success } \\
\text { (active state), } & \\
\text { est_c }, & \text { if slot } k \text { had one collision } \\
\text { (backlog state) },
\end{array}\right.
$$

where est_i, est_s, est_c are defined as:

$$
\begin{aligned}
& \text { est_i }=2-e, \\
& \text { est_s }=2-\frac{e}{1-p_{e}}, \\
& \text { est_c }=2 .
\end{aligned}
$$

where $p_{e}$ is the probability of the packet not being accepted by the receiver.

With these values, it is possible to calculate in a dynamic form the estimate, Equation (8), obtaining control of the load of the system. Therefore, the objective is to operate with a binary exponential retransmission algorithm [14] that permits us to stabilize the response of S-Aloha, as it will be seen in Section 3.

\section{CONCLUSIONS}

In this work, we have modeled and simulated a RACH with S-Aloha, considering a real environment. To increase the efficiency of the system, the capture effect was used with a uniform spatial distribution and the presence of the channel. This paper has demonstrated that spatial distribution of the MTs enables capture effect while providing SAloha the capability to substantially improve system throughput. In addition, this paper has shown that the superimposed Rayleigh fading, shadowing, and spatial distribution of the MTs can further enhance the effect capture, resulting in S-Aloha with higher throughput.

Simulation results have indicated that system performance is not sensitive to Rayleigh fading for a given BS receiver sensitivity, and that S-Aloha performance is improved by reducing capture ratio. The spatial distribution gives nearby MTs a further increased probability to survive a collision in contrast to a channel with Rayleigh fading, where the probability that an individual signal is sufficiently strong to survive a collision is very small. Simulation results have also indicated that when using the algorithm of dynamic retransmission with the feedback ternary information of the channel, the throughput system does not decrease as the channel load increases, and the system delay is reduced. This occurs because the retransmission algorithm optimizes channel management and enables the system to quickly resolve data packet collisions.

\section{REFERENCES}

[1] Zhou, H and Deng, R. H, "Capture Model for Mobile Radio Slotted ALOHA Systems", IEE Proceedings Communications., Vol. 145, No. 2, April 1998, pp. 91-97

[2] Zorzi, M. and Rao, R. R., "Capture and Retransmission Control in Mobile Radio," IEEE Journal on Selected Areas in Communications, Vol. 12, No. 8, October 1994, pp. 1289-1298.

[3] Bertsekas, D. and Gallager, R., Data Networks, Prentice-Hall, 1992.

[4] Bottcher, A. and Dippold, M., "The Capture Effect in Multiaccess Communications-the Rayleigh and Landmobile Satellite Channels," IEEE Transactions on Communications, Vol. 41, No. 9, September 1993, pp. 1364-1372.

[5] Habbab, I., Kavehrad, M., and Sundberg, C., "ALOHA with Capture Over Slow and Fast Fading Radio Channels with Coding and Diversity," IEEE Journal on Selected Areas in Communications, Vol. 7, No. 1, January 1989, pp. 79-88. 
[6] Covarrubias, D., et. al., "An efficient Adaptive Coding Scheme for Data Transmission over a Fading and Nonstationary Mobile Radio Channel," Proceedings of the IEEE $3^{\text {rd }}$ International Symposium on MultiDimensional Mobile Communications-MDMC'98, September 1998, pp. 168-173.

[7] Taylor, H. M. and Karlin, S., An Introduction to Stochastic Modeling. Academic Press Inc., 1994.

[8] Covarrubias, D., Procedures and Techniques of Dynamic Assignment and Stabilizing of Applicable MAC to Mobile Systems of Third Generation (in Spanish). Ph.D. Thesis. UPC, Spain, 1999.

[9] Covarrubias, D., et.al., "Spatial Distribution Analysis with Capture Effect of a Mobile S-Aloha Network," Proceedings of the IEEE International Symposium on Personal, Indoor and Mobile Radio Communications, vol. 3, 1998, pp. 1116-1120.

[10] Rappaport, T.S., Wireless Communications: Principles and Practice, 1 1" ed., U.S.A.:Prentice Hall PTR, 1996.

[11] Linnartz, J. P. and Prasad, R., "Near-Effect on Slotted Aloha Channel with Shadowing and Capture," Proceedings of the IEEE Vehicular Technology Conference'89, 1989, pp. 809-813.

[12] Van Der Plas, C. and Linnartz, J. P., "Stability of Mobile Slotted ALOHA Network with Rayleigh Fading, Shadowing, and Near-Far," IEEE Transactions on Vehicular Technology, vol. 39, No. 4, November 1990, pp. 359366.

[13] Rivest, R. L., "Network Control by Bayessian Broadcast," IEEE Transactions on Information Theory, vol. 33, No. 3, May 1987, pp. 323-328.

[14] Mendez, A. and Covarrubias, "Stability and Optimal Retransmission Control of S-Aloha as a RACH Channel on Wireless Networks," Proceedings of the 54 ${ }^{\text {th }}$ IEEE Vehicular Technology Conference, vol. 3, October 2001, pp. 1368-1372.

Authors Biography

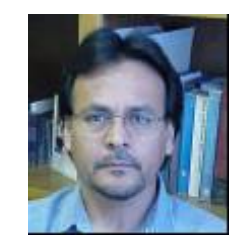

\section{David Covarrubias.}

Obtained his BS in engineering from the Universidad Nacional Autónoma de Mexico (UNAM), México, MSc from CICESE, Ensenada Baja California, Mexico and his PhD (cum laude) from the Universitat Politecnica de Catalunya (UPC), Barcelona, Spain, all in telecommunications area. He has been member of the scientific staff of Electronics and Telecommunications department at CICESE since 1984. His current research interests include smart antennas, MAC and scheduling modeling for 3G mobile communications. He is a member of the Sistema Nacional de Investigadores (SNI) of México

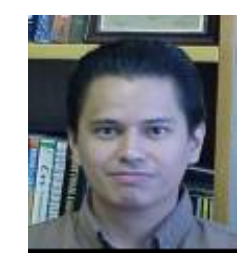

\section{Aldo Mendez}

Obtained his BS in engineering from the Universidad Veracruzana, Veracruz, Mexico in 1995, MSc from CENIDET, Cuernavaca, Morelos, Mexico in 1997 and his PhD from the CICESE, Ensenada, Baja California, Mexico in 2003. He has been member of the scientific staff of Electronics and Telecommunications department at UAT since 2004. His current research interests include MAC and scheduling modeling for mobile communications. He is a member of the Sistema Nacional de Investigadores (SNI) of México 


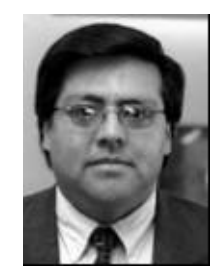

Cesar Vargas Rosales

Received the B.Sc. degree in Mechanical and Electrical Engineering from the National Autonomous University of Mexico (UNAM) in 1988, the M.Sc. and the Ph.D. in Electrical Engineering from Louisiana State University (LSU), Baton Rouge, Louisiana, U.S.A. in 1992 and 1996, respectively. Since 1996, he has been in the Center for Electronics and Telecommunications at ITESM-Campus Monterrey, Monterrey, Mexico where he is an Associate professor. His main research interests are on Personal Communications Networks, mobility modeling, traffic modeling, multiple accesses and scheduling in wireless networks, routing and QoS in wireless ad-hoc networks. He is a member of the Sistema Nacional de Investigadores (SNI) of Mexico. 